\title{
Blind Equalization Algorithm for 16-QAM Signals
}

\author{
Jianping ZHANG, Xiaoling ZENG \\ Chongqing Creation Vocational College,Yongchuan,Chongqing \\ amybaoer@126.com, b43687961@qq.com
}

\begin{abstract}
Keywords: Modify Constant Modulus Algorithm(MCMA), Decision-Directed Least-Mean Square(DD-LMS), Blind Equalization Algorithm, Joint Equalization

Abstract. This article propose and use the Modify Constant Modulus Algorithm (MCMA) and Decision-Directed Least Mean Square (DD-LMS) joint equalization Algorithm to balance Inter-Symbol-Interference (ISI) at the receiving end of 16-QAM. We analyzed the joint equalization technology implementation and switching threshold. Also we had carried on simulation experiments of four different equalization algorithms. The simulation results show that MCMA and DD-LMS joint equalization algorithm can get smaller residual error iteration, also can effectively repair the transmission process of phase shift.
\end{abstract}

\section{Introduction}

In the continuous developing communication field, it is necessary to improve the transmission rate of information as the amount of information becomes larger. We can improve the signal processing hardware and software of the communication transmitter and receiver to improve the transmission rate of information. Also we can adopt a higher code system ${ }^{[1]}$. The modulation method based on the 16Quadrature Amplitude Modulation(16QAM) constellation diagram can provide a high code rate of modern communication system which meets the needs of modern communication development. In the receiving end, the equalization technique is used to eliminate the interference between codes.

The MCMA algorithm has the advantages of high convergence performance, low computational complexity of equalizer and simple structure ${ }^{[2]}$. However, the equalization algorithm has low convergence speed and large residual error while the Decision-Directed Least-Mean Square(DD-LMS) algorithm has a lower residual error. When the error is to large at the beginning of the balance, the DD-LMS algorithm may judge error which will cause residual error divergence. So it is undesirable to directly use the DD-LMS algorithm to make the equalizer cold start ${ }^{[3]}$. If we just use one of the above equalization algorithms, it is unable to adapt to the demand of the communication signal processing with growing development. It greatly restricts the effectiveness of the equalizer, and the 16QAM signal processing has great limitations.

In this paper, MCMA and DD-LMS algorithm are used to combine blind equalization so that the equalizer simultaneously have the advantages of error convergence and small residual error after equalizing.

\section{Joint equalization algorithm}

In the joint equalization, while the using of the CMA/MCMA and DD-LMS equalization algorithms is determined, it is important to determine when to switch the two algorithms. If the switch is too early that the CMA/MCMA has not converged, the error rate will be so high that the DD-LMS algorithm can not converge. This will cause the divergence of equalizer and a high error rate. But if the switch is too late, it is easy to cause the convergence speed slow. Therefore, we must determine an optimal threshold switching time.

Blind equalization algorithm can be divided into two modes : replenishment and follow-up tracing. The CMA algorithm has robust convergence which can be used to make the eye open at the beginning of equalization. After opening the eye, the DD-LMS algorithm can be used to decrease the residual error ${ }^{[4]}$. 
The basic idea of joint equalization is using CMA or MCMA algorithm as cold start when the channel eye is not opened. Through a certain number of iterations, the DD-LMS algorithm can be switched when the error rete is low, which can make the residual error further reduced.Figure 1 is the 16QAM constellation diagram of communication system. When the residual error $e(n)=z(n)\left(|z(n)|^{2}-R_{2}\right)$ of the CMA or MCMA algorithm has not converged to the eye opening, residual error has not reached the switching threshold, we should continue to use CMA or MCMA algorithm to converge till the judgment makes the eye open and most of the points falls in the small circles in Figure 1. And at this time, switch to the DD-LMS algorithm that can speed up convergence and reduce residual error.

In addition, at the beginning of early convergence iterative of equalization, there may iterative to small circles in Figure 1. But the eye is not open actually. It can not meet the requirements of the DD-LMS algorithm. Therefore, the improved algorithm will be several times prohibited to switch to the DD-LMS algorithm before the start of the iteration.

Assuming that $n(n)$ is the channel Gauss white noise, the variance is $\sigma^{2}$, the mean value is 0 , thus signal to noise ratio of the output signal in communication system is:

$$
S N R=10 \lg \frac{\mathrm{E}\left[x(n)^{2}\right]}{\sigma^{2}}
$$

In the form, $\mathrm{E}\left[x(n)^{2}\right]$ is the power of the sending end signal.

Assuming that $R_{\max }$ is the outer signal radius of the signal constellation diagram sended by 16QAM signal. Thus the noise standard deviation can be derived by form (1):

$$
\sigma=\frac{R_{\max }}{\sqrt{10^{S N R / 10}}}
$$

Therefore, the circle which take the constellation point in constellation diagram as the center of the circle and $\sigma$ as the radius of the circle, is the decision point after the equalizer is convergent. So the joint equilibrium algorithm which take this as the switching threshold circle of the decision circle can be used to verdict, as shown in Figure 1.

At the same time, assuming that the minimum Euclidean distance of the 16QAM constellation diagram is $d$. Then to meet the convergence performance of the decision algorithm, the range of the convergent circle's radius $r$ is:

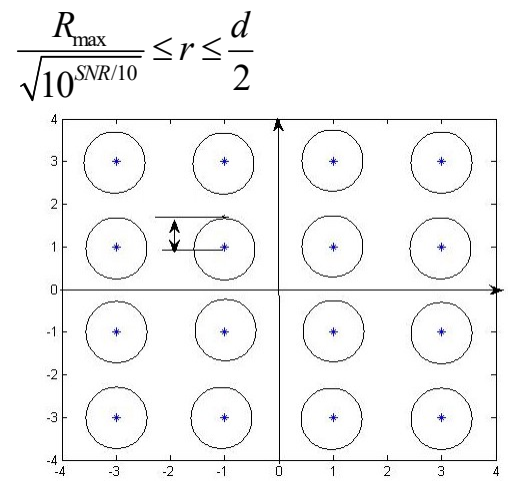

Fig. 1 the decision of switching to the DD-LMS algorithm

In the joint equilibrium, the iterative formula of the tap coefficient is as follows:

$$
\left\{\begin{array}{l}
\mathbf{u n} \\
W(n+1)=\underset{W}{\mathbf{u}}(n)-\mu_{C M A / M C M A}^{\prime} e_{C M A / M C M A}(n) Y^{\mathbf{u}}{ }^{*}(n), z(n) \notin D \\
\mathbf{u n} * \\
W(n+1)=W(n)-\mu_{D D-L M S}^{\prime} e_{D D-L M S}(n) Y^{*}(n), z(n) \in D
\end{array}\right.
$$

In the upper form, $D$ is the small circle area of Fig.1.

When the calculation results of the error have not converged to the small circle in Figure 2, we can use the first formula in form (4) and use the CMA or MCMA algorithm to do the error iterative calculation. When the calculation results of the error have converged to the small circle, we can use 
form 2 to update the tap coefficient by using the DD-LMS algorithm. Through switching the two kinds of error calculation formula, we can achieve joint equalization.

\section{Simulation results and analysis}

In order to verify the proposed joint equalization algorithm, we compare the CMA algorithm, the MCMC algorithm, the joint equalization algorithm of the CMA and DD-LMS algorithm with the joint equalization algorithm of the MCMA and DD-LMS algorithm. We compare the effects of convergence and equalization constellation diagram caused by all kinds of equalization algorithms. Also we compare and analysis the residual error of all kinds equalization algorithms.

The simulation parameters are set up as follows: the channel response function $\mathrm{h}=[-0.005-0.004 \mathrm{j}$ $0.009+0.030 \mathrm{j}-0.024-0.104 \mathrm{j} 0.854+0.520 \mathrm{j}-0.218+0.273 \mathrm{j} 0.049-0.074 \mathrm{j}-0.019+0.020 \mathrm{j}]$; noise signal is the Gauss white noise, the signal-to-noise ratio $\mathrm{SNR}=30 \mathrm{~dB}$; the iterative step of the DD-LMS algorithm $\mu \mathrm{DD} \neg \mathrm{LMS}=0.005$; signal sequence length $\mathrm{N}=5000$; the minimum Euclidean distance $\mathrm{d}=2$; the number of tap coefficients $\mathrm{L}=13$; The minimum number from the CMA/MCMA algorithm to the DD-LMS algorithm is Num=80; The error threshold value from the CMA/MCMA algorithm to the DD-LMS algorithm is threshold=0.6. Simulation effect diagram is as follows:
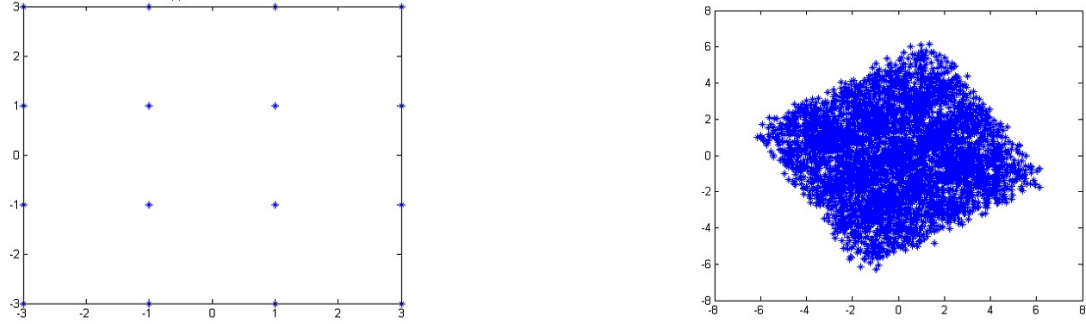

Fig. 2 16QAM Encoding Modulation Constellation Fig.3 Constellation after channel transmission

Shown as Figure 2, 16QAM constellation diagram is $4 * 4$ constellation points. The experimental set minimum Euclidean distance is 2. But in Figure 3, the channel has switched, and it has added the receiving end signal sequence when the signal-to-noise ratio $\mathrm{SNR}=30 \mathrm{~dB}$. We can see that the deformation of the receiving end signal and the error rate are large. Therefore, only through the equalizing can restore the signal of the sending end.

\section{Comparison and analysis of equalizer output constellation diagram}

Doing simulations to the CMA algorithm, the MCMA algorithm, the joint equalization algorithm of the CMA and DD-LMS algorithm, the joint equalization algorithm of the MCMA and DD-LMS algorithm, the obtained equalization output the constellation diagram, shown as Figure 4.

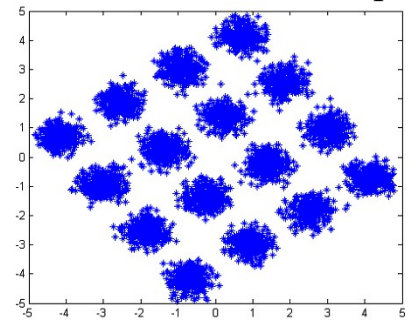

(a) CMA

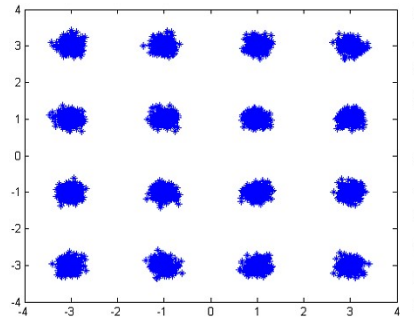

(b)MCMA

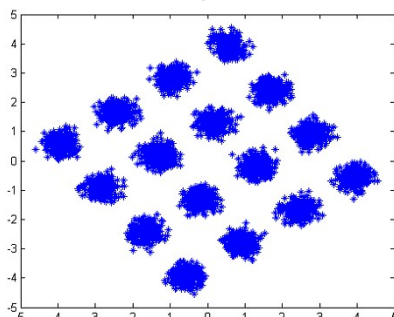

(c) CMA and DD-LMS

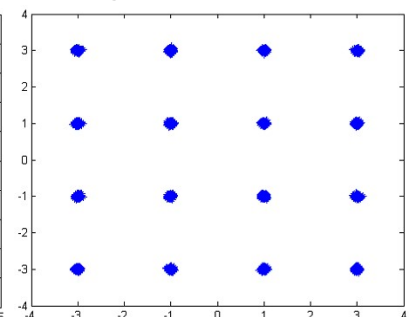

(d)MCMA and DD-LMS

Fig. 4 the equalizing effect diagram of each algorithm

From Fig.4 (a), after equalizing by the CMA algorithm, the signal sequence of the receiving end can basically convergence which can make the eye open. The CMA algorithm has advantages of robust convergence performance and low computational complexity. However, from the Figure, we can see that the signal sequence equalized by the CMA algorithm is divergent and has phase shift. It is very disadvantageous to the subsequent decision processing.

From Fig.4 (b), we can see that the MCMA algorithm has already eliminated the phase shift problem in the CMA algorithm. The separate estimation makes the error estimation smaller. Thus the point in the Figure4 (b) is more convergent than the point in the Figure4 (a). Fig.4(c) is the effect diagram of the joint equalization of the CMA and DD-LMS algorithm. To make comparison with 
Figure4 (c) and Fig.4 (a), we can see that the constellation point equalized by 16QAM can further converge after joint equalization. This also makes the residual error smaller. However, the joint equalization of the CMA and DD-LMS algorithm can not solve the problem of phase defection. The Fig.4(d) is the output result adopting the joint equalization algorithm of the MCMA and DD-LMS algorithm. In Fig. 4(d), each constellation point has converged very small, which achieves the optimal equalization effect of all above equalization algorithms.

In the equalization simulation, two constants are used to set the threshold at the same time when the threshold is switched from CMA/MCMA to DD-LMS algorithm. First, it is stipulated by he simulation experiment that first 80 iterations which is starting from tap coefficients must use the CMA/MCMA algorithm. The reason is that the equalization may meet the switching threshold of the DD-LMS algorithm by coincidence at the beginning. But at this time, the equalization has not converge to a small enough convergence value. If the DD-LMS algorithm is enabled by coincidence, it may cause the divergence of the equilibrium effect. In order to avoid this situation and increase the stability of the iterative system, a constant is set.

$$
\begin{gathered}
e(n)=z(n)\left(|z(n)|^{2}-R_{2}\right) \\
e(n)=e_{R}(n)+e_{I}(n)=z_{R}(n)\left(\left|z_{R}(n)\right|^{2}-R_{2, R}\right)+z_{I}(n)\left(\left|z_{I}(n)\right|^{2}-R_{2, I}\right)
\end{gathered}
$$

When the threshold value is switched from the CMA/MCMA to the DD-LMS algorithm, the CMA error function definition formula (5) and the residual error judgment formula (6) are adopted. When the error of the two formulas is less than 0.6, the DD-LMS algorithm is switched. This all achieve the switching between the two equalization algorithms.

\section{Comparison and analysis of residual error}

After doing 100 repeated experiments of 5000 signal samples each time for each of the four equalization algorithms, calculate the residual iterative error of each experiment. As the Figure 5 shown, all of the four algorithms make the residual error decrease rapidly after iterating over 100 signal sequences, but the residual error is very large finally. The joint equalization of the MCMA and DD-LMS algorithm has a small residual error.

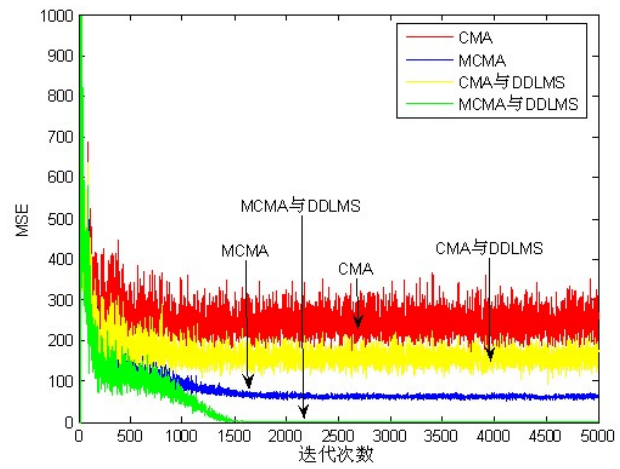

Fig.5 equalization algorithm residual error graph

\section{Conclusions}

In this paper, the performance of various equalization algorithm and joint equalization algorithm are verified by simulation. From this we can see that joint equalization algorithm has a better equilibrium effect and smaller residual error than the CMA algorithm. It can be seen intuitively that the constellation point of the joint equalization algorithm is very convergent.

\section{Acknowledgment}

We thank our colleagues for providing insights, support, and suggestions that improved this work. 


\section{References}

[1] Wen-Tsai Sung, Implementation of enhanced fractionally spaced algorithm for blind equalization technology in WSNs: Ad Hoc Networks. Vol. 14 (2014), p. 26-34

[2] J.E. Araújo: Dithiothreitol-based protein equalization technology to unravel biomarkers for bladder cancer, Talanta, 2017

[3] Wen-Tsai Sung,Wireless sensing network transmission system with improved constant modulus algorithm, EURASIP J. Wireless Commun.Netmork. Vol. 101 (2013), p. 1-8

[4] Fei Xu and Yaojun Qiao, Laser linewidth and fiber nonlinearity tolerance study of C-16QAM compared to square 16QAM in coherent OFDM system, Optical Fiber Technology.(2017), p. 36-41 